\title{
Thermosensitive Hollowed Magnetic Lignin Nanocarrier Laden with Doxorubicin for Responsive Delivery and Hyperthermia Treatment of Cancer
}

\author{
Anyik John Leo, Frans B Waanders, Martin Mkandawire and Elvis Fosso-Kankeu
}

\begin{abstract}
To improve effectiveness while using very low doses to avoid side effects on one hand and maintain affordability, we are proposing a therapeutic system that can target cancerous cells with more precision and deliver the drug upon induction by the doctor. The concept is to use magnetised polymer-based nanocapsules of cancer drugs, which can be magnetically direct to the targets and respond to magnetic stimulus and hyperthermia as well as magnify the drug potency. Hence, we have developed: (a) an affordable self-assembly method of synthesizing lignin hollowed nanoparticles with high loading capacity for the encapsulation of doxorubicin drug molecules; and (b) a method of magnetising the lignin polymer and surface functionalised on the nanocapsule with a thermosensitive agent. Upon exposure to an external magnetic field, the cancer cells will be selectively targeted, and the generation of heat will induce the release of the drug molecules from the capsule and amplify the potency of doxorubicin drug that even low doses become effective.
\end{abstract}

Keywords - apoptosis, drug delivery, magnetic nanoparticles polymeric nanoparticles, tumour

\section{INTRODUCTION}

Cancer is unarguably the most vicious affliction that destroys millions of lives worldwide and is characterised by a group of defective cells known as tumours arising from several gene mutations[1]-[2]. These abnormal cells have introduced a complex and acidic microenvironment ( $\mathrm{pH}$ range $6.0-6.8$ ) to protect and support their activities and tend to develop survival mechanisms against any unusual intervention to hinder their co-existence. The buffering, alkalinization or anti-acidic treatment of the cancer cells will selectively change its microenvironment by destroying their metabolic machinery

Manuscript received September 1, 2020. This work was supported in part by North West University and Cape Breton University.

A. John Leo is with is with (1). The Water Pollution Monitoring and Remediation Initiatives Research Group, School of Chemical and Minerals Engineering, North-West University, Potchefstroom, South Africa. (2). The Solid-state Research Group, Department of Chemistry, School of Science and Technology, Cape Breton University, Canada.

F.B. Waanders is with the Water Pollution Monitoring and Remediation Initiatives Research Group, School of Chemical and Minerals Engineering, North-West University, Potchefstroom, South Africa

E. Fosso-Kankeu is with the Water Pollution Monitoring and Remediation Initiatives Research Group, School of Chemical and Minerals Engineering, North-West University, Potchefstroom, South Africa.

M. Mkandawire is with the Department of Chemistr, School of Science and Technology, Cape Breton University, Canada. and starving the cells to death[3]-[4]. Clinically, chemotherapy is the most reliable treatment option used over the last decades to provide great hope for cancer patients. Chemotherapeutic agents such as Doxorubicin(Dox) is the most affordable medication approved by the Food and Drug Administration (FDA) as a frontline treatment for cancer therapy. Despite its potential to induce tumour cell apoptosis, it is far from being perfect[5]-[7]. Long-term use of Dox can dose-relatedly cause cardiotoxicity in a patient and the risk of cardiotoxicity intensify in elderly people[8]. Eventually, an elderly cancer patient with a weak heart is excluded from using Dox[9]. Dox also lacks the selectivity towards cancerous cells, and therefore, the drug accumulation at tumour sites is usually low.

Consequently, higher drug doses are required for treatment which may accumulate in healthy cells producing undesirable side effects such as infertility, hepatotoxicity, nephrotoxicity impacting the patient quality of life[10]-[12]. The resulting side effects also incurred additional costs besides compromising with the patient's quality of life. For instance, young cancer patients reproductive cells are often collected and well-preserved before inducing them with Dox for later fertility treatment[9].To overcome Dox's impediment, it is imperative to minimize its toxicity and increase its therapeutic effect against cancer cells that even low doses become effective. Several research studies have been conducted to enhance the chemosensitivity of Dox to reduce its sides effects. To name a few, Li et al reported the combination of a cardioprotective agent Tanshinone IIA(Tan IIA) with Dox as an innovative agent for breast cancer therapy[13]. Tan IIA not only enhances the chemotherapeutic effect of Dox against breast cancer but protects against cardiotoxicity. In another study, Chen and co-workers combine Dox with Dioscin and the results showed that dioscin significantly ameliorate Dox-induced cell injury, reduced reactive oxygen species (ROS) level, and suppressed cell apoptosis in alpha mouse liver cells. However, these studies did not guarantee that the in vivo application of the combination therapy will be able to accumulate Dox on the disease sites, and to blind the body's defence mechanism and the reticuloendothelial system not to recognize the therapeutic agent as foreign material and remove it from the bloodstream before reaching the targeted site. An advance approach to improving doxorubicin's efficiency is to modify its pharmacokinetics and tissue distribution using a targeted drug delivery system to effectively transport and discharge the 
medication at the disease sites[14]. The emergence of advanced nanotechnology has revolutionized the biomedical field and eventually, unwrapped possibilities for creating nanocapsules as targeted drug delivery systems to give new life to drugs with a low therapeutic index. Such technological innovation has yielded the potential to incorporate customized therapeutic agents, controlled-release mechanisms, and targeting strategies within a single nano-scaled architecture for disease therapy[12],[15]. The combined functionality within a single nanosystem for cancer therapy is efficient circumventing single-drug systemic administration limitations such as poor bioavailability of drug at tumour sites, drug resistance and rapid renal clearance[16]. Amazing studies have been conducted to ensure Dox's safe delivery at the tumour sites using the various customized delivery systems, and details of this will be discussed below. However, these customized systems also face some limitations which must encourage us to develop a therapeutic system that can target cancerous cells with more precision and deliver the drug upon induction by the doctor.

This mini-review highlights a systematic approach to developing a highly effective hybrid thermo-chemotherapeutic system for tackling cancerous tissue.

\section{TYPES OF NANOCARRIERS USED FOR DOXORUBICIN DELIVERY AND THEIR LIMITATIONS}

Over the last decade,many research efforts have been invested in developing targeted drug delivery in the nanoscale to deliver medication to tumours or cancerous cells. Despite some advancements, targeting the medication to the tumour or cancerous cells has not yielded much success, partly due to reasons including body response to the drug-delivery system, the untimely release of the drug, and cytotoxicity of the drug-carrying system. Various nanocarriers(scheme 1) have been laden with chemotherapeutic drugs by chemical conjugation or physical entrapment and explored for cancer therapy. Although most of them have reached the clinical stage, they still have shortcomings that diminish their efficiency.
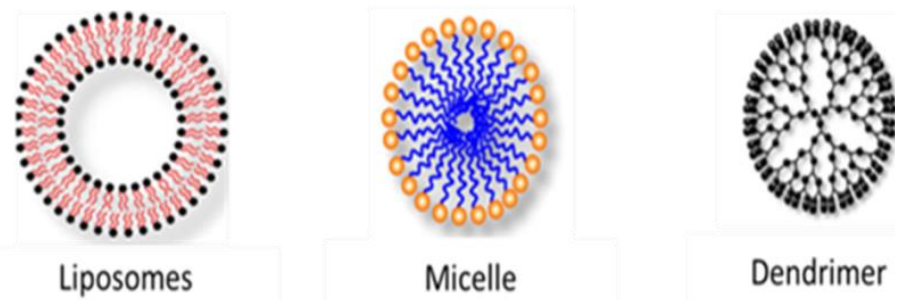

Dendrimer

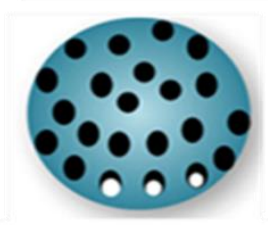

Mesoporous

Scheme 1. Types of nanocarrier platforms used for drug delivery

\section{A. Liposomes nanocarrier}

Liposomes are phospholipid vesicles in nature that have been clinically approved as a nanocarrier for their unique ability to entrap both lipophilic and hydrophilic compounds within their network and channel them to the required diseased site[17]-[18]. Liposomes laden with doxorubicin have been effectively used for cancer therapy to improve the pharmacokinetic-profile and pharmacological properties of doxorubicin drug[19]. For example, liposomes loaded with Dox and surface functionalized with a pegylated agent( Doxil@) was approved in 2005 for ovarian cancer. However, this delivery system still faces some limitations which compromise their effectiveness. Since liposomes are phospholipids in nature and, when administered intravenously, the body's defence mechanism, including the reticuloendothelial system (RES), recognises it as foreign material and eventually, it is being cleared and removed from the system before reaching the targeted sites[20]. This is attributed to the fact that lipid-based nanocarriers such as liposomes resemble the size and shape of pathogenic microorganisms of which the innate immune system happens to target[21].

\section{B. Micelles and Dendrimers nanocarrier for drug delivery}

Polymeric nanocarriers such as micelles and dendrimers have also been loaded with Dox and surface functionalized with targeting moiety for clinical trials. Micelles are self-assembled colloidal particles consisting of a hydrophilic head and a hydrophobic tail capable of enhancing the solubility, permeability and bioavailability of drugs due to their unique features reaching higher concentration in the biological system[22]. While dendrimers are highly symmetrical hyperbranched polymers with tree-like structures consisting of a central core and more than two branches with terminal functional groups[23]. They have the potential to omit biological problems and to reach the right targets such as the first-pass effect, immune clearance, cell penetration, and off-target interactions[24]. Despite the potential advantages of these polymeric systems as nanocarriers, they still face some limitations such as the low loading capacity of drug molecules which compromise their efficiency.

\section{Mesoporous Silica Nanoparticles}

Inorganic mesoporous silica nanoparticles (MSNs) have attracted much attention for diagnostic and therapeutic applications due to their large surface area and pore volumes which offer high loading capacity to absorb and carry other therapeutic compounds via encapsulation or surface attachment[25]-[28]. MSNs also have good chemical stability, good biocompatibility, and low toxicity which are easy to obtain and cheap to produce. Additionally, their high specific surface area with abundant $\mathrm{Si}-\mathrm{OH}$ active groups on the pore walls will ensure the effective bonding of other organic functionalities that will act as a gatekeeper to prevent the premature release of loaded drugs and targeting ligands[15],[26]. However, MSNs also have their shortcomings which limit their application in the biomedical field. For instance, MSNs are rapidly cleared from the blood circulation system as the phagocytic cells in the liver and spleen recognize them as foreign materials[29]. 


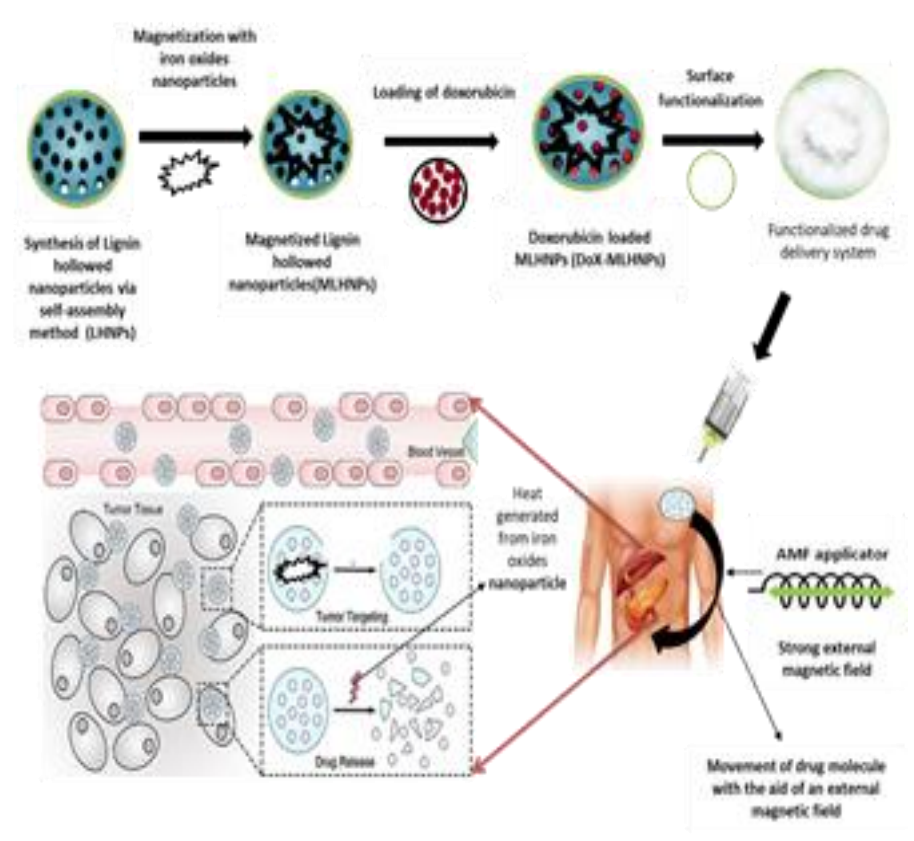

Scheme 2. Schematic representation of magnetised lignin hollowed nanoparticles for drug delivery and hyperthermia treatment of for cancer

\section{TARGETED DRUG DELIVERY SYSTEM}

The efficiency of chemotherapy can be improved if the drug only targets the disease cells and avoids the uncontrolled distribution into healthy tissues, causing most of the undesired sides effect. Incorporating magnetization into nanoparticles with high loading capacity that responds to a strong external magnetic field is emerging as an innovative drug delivery strategy. In this regard, magnetic nanoparticles embedded in polymer material are being investigated as a potential system to directly transport drug molecules into disease cells to concentrate this chemotherapeutic to the affected region under the influence of an external magnetic field[30]. The induction of an external magnetic field will also cause the system to generate heat in a hyperthermia process, which further assists in killing the disease cells[31]. The heat generated can increase the cell's temperature from 39 to $45 \mathrm{oC}$, which stimulates cell stress, which denatures the cell protein leading to death[32]. The co-delivery of drug molecules and heat in the disease cells will synergistically exhibit high therapeutic effects with a very low dosage of the drug molecule. Magnetic drug delivery system (MDDSs) is revolutionising the field of drug delivery because of its biocompatibility and its potential use as a tracking, targeting and drug-delivery system for cancer diagnosis and therapy[33]. ]. As such, several research studies have been conducted on the development of these systems. In a recent study, incorporating superparamagnetic iron oxide nanoparticles with polyaspartamide (PA) biopolymer laden can reduce the growth of cancerous cells by three times compared to that of uninjected controls[34]. In another attempt to test for anticancer activity, Jahanban-Esfahlan and co-workers were able to develop a magnetic natural hydrogel delivery system and laden with doxorubicin in which the system was able to induce cytotoxicity on Hela cells. Ayyanaar et al. also made contributing progress where they reported the synthesis of magnetised chitosan nanoparticles loaded with 5-fluorouracil, an anticancer agent[35]. The magnetised delivery system induced cytotoxicity and inhibited the formation of colonies for A549 and HeLa S3 cancer cells. The positive outcome of these developed magnetized drug delivery systems suggested its use to overcome chemotherapy's dilemma, but the shortcoming is the availability, loading efficiencies, and affordability of the delivery system. Therefore, our research group is developing a magnetized delivery system with a cost-effective polymer with a high loading capacity. This is a novel study that has not been reported, and the envisaged technology will be based on two well-established techniques, namely (1) fabrication of biopolymeric hollowed nanoparticles and (2) magnetization of the nanocarrier and surface functionalization.

\section{Fabrication of polymeric hollowed nanocarrier}

Polymeric nanocarriers such as liposomes, dendrimers or micelles represent an amazing avenue for the encapsulation or delivery of drugs in cancer therapy as these systems are biodegradable, biocompatible, non-toxic with a high loading capacity for therapeutic agents[36]. As aforementioned, these polymeric nanocarriers have some limitations which compromised their applications in cancer therapy. Lignin is an exceptional polymeric material with unique properties and is the second-most abundant organic polymer after cellulose[37]. Lignin is non-toxic and structurally. It contains phenolic and aliphatic hydroxyl groups that can be modified through diverse chemical reactions into lignin hollowed nanoparticles (LHNPs) for drug delivery[38]. Lignin nanoparticles have been previously synthesized through sonication and precipitation methods, but the limitations of these methods are toxic reagents, limiting their applications in the biomedical field. Recently, Xiong et al. reported a straightforward self-assembly method for synthesizing lignin hollowed nanoparticle by dissolving enzymatic hydrolysis lignin in tetrahydrofuran while dropping deionized water to the lignin/tetrahydrofuran mixture[39]. Tetrahydrofuran is used as a surfactant/template and is being removed in the process, making this method a safe and clean process for obtaining lignin hollowed nanoparticle.

\section{The magnetization of nanocarrier for cancer therapy and surface coating}

The targeting of disease sites using a magnetic delivery system is an effective approach in nanomedicine, and materials used for inducing the magnetic behaviour are usually colloidal iron oxides nanoparticles with a superparamagnetic property[40]. The application of Iron oxides nanoparticles has been approved by the Food and Drug Administration(FDA) and in clinical use for decades because of its safety[41]. Therefore, incorporating iron oxides nanoparticles with polymeric nanocapsules will present a novel drug delivery system with excellent biological features for enhanced and targeted drug delivery for cancer therapy. Upon loading doxorubicin into the magnetic nanocarrier, the delivery system's surfaces are coated with materials such as hydrogel to avoid premature release when injected into the vascular system. The hydrogel material will respond to physicochemical properties such as $\mathrm{pH}$, light or temperature to enable the drug's release at the targeted sites. 


\section{CONCLUSION}

Polymeric magnetic nanoparticles loaded with chemotherapeutics such as doxorubicin is a novel avenue for cancer therapy. Once present in the respective cancer cells' supplying vascular system and exposed to an external magnetic field, the cancer cells will be selectively targeted, and higher doses of the chemotherapeutic agent will be concentrated at the disease sites. The heat generated due to the hyperthermia effect in the process and with the drug molecules will synergetically kill the cancer cells. Eventually, healthy cells will not be affected and will improve patient health after successful therapy.

\section{ACKNOWLEDGEMENT}

The authors are grateful of the financial support from the North-West University.

\section{REFERENCES}

[1] Li, X., Taratula, O., Taratula, O., Schumann, C. and Minko, T., 2017. LHRH-targeted drug delivery systems for cancer therapy. Mini reviews in medicinal chemistry, 17(3), pp.258-267.

https://doi.org/10.2174/1389557516666161013111155

[2] Jeetah, R., Bhaw-Luximon, A. and Jhurry, D., 2014. Polymeric nanomicelles for sustained delivery of anti-cancer drugs. Mutation Research/Fundamental and Molecular Mechanisms of Mutagenesis, 768, pp.47-59.

https://doi.org/10.1016/j.mrfmmm.2014.04.009

[3] Ahmed, N., Escalona, R., Leung, D., Chan, E. and Kannourakis, G., 2018 , October. Tumour microenvironment and metabolic plasticity in cancer and cancer stem cells: Perspectives on metabolic and immune regulatory signatures in chemoresistant ovarian cancer stem cells. In Seminars in cancer biology. Academic Press.

https://doi.org/10.1016/j.semcancer.2018.10.002

[4] Logozzi, M., Mizzoni, D., Angelini, D., Di Raimo, R., Falchi, M., Battistini, L. and Fais, S., 2018. Microenvironmental pH and exosome levels interplay in human Cancer cell lines of different Histotypes. Cancers, 10(10), p.370. https://doi.org/10.3390/cancers10100370

[5] Sun, L., Chen, Y., Zhou, Y., Guo, D., Fan, Y., Guo, F., Zheng, Y. and Chen, W., 2017. Preparation of 5-fluorouracil-loaded chitosan nanoparticles and study of the sustained release in vitro and in vivo. Asian journal of pharmaceutical sciences, 12(5), pp.418-423. https://doi.org/10.1016/j.ajps.2017.04.002

[6] Dou, X.Q., Wang, H., Zhang, J., Wang, F., Xu, G.L., Xu, C.C., Xu, H.H., Xiang, S.S., Fu, J. and Song, H.F., 2018. Aptamer-drug conjugate: targeted delivery of doxorubicin in a her3 aptamer-functionalized liposomal delivery system reduces cardiotoxicity. International journal of nanomedicine, 13 , p.763. https://doi.org/10.2147/IJN.S149887

[7] Senapati, S., Mahanta, A.K., Kumar, S. and Maiti, P., 2018. Controlled drug delivery vehicles for cancer treatment and their performance. Signal transduction and targeted therapy, 3(1), p.7. https://doi.org/10.1038/s41392-017-0004-3

[8] Zhao, L.; Zhang, B., Doxorubicin induces cardiotoxicity through upregulation of death receptors mediated apoptosis in cardiomyocytes. Scientific Reports 2017, 7 (1), 44735 https://doi.org/10.1038/srep44735

[9] Qiao, X., van der Zanden, S.Y., Wander, D.P., Borràs, D.M., Song, J.Y., Li, X., van Duikeren, S., van Gils, N., Rutten, A., van Herwaarden, T. and van Tellingen, O., 2020. Uncoupling DNA damage from chromatin damage to detoxify doxorubicin. Proceedings of the National Academy of Sciences, 117(26), pp.15182-15192. https://doi.org/10.1073/pnas.1922072117

[10] Chen, X., Zhang, B., Song, S., Chu, L., Liang, H., Chen, J., Liang, J. and Huang, Z., 2019. Protective effects of dioscin against doxorubicin-induced hepatotoxicity via regulating Sirt1/FOXO1/NF-кB signal. Frontiers in Pharmacology, 10, p.1030. https://doi.org/10.3389/fphar.2019.01030
[11] Leung, J., 2018. Recent Advances in Nanoparticle-Based Cancer Drug Delivery.

[12] Alexis, F., Pridgen, E., Molnar, L.K. and Farokhzad, O.C., 2008. Factors affecting the clearance and biodistribution of polymeric nanoparticles. Molecular Pharmaceutics, 5(4), pp.505-515. https://doi.org/10.1021/mp800051m

[13] Li, L., Wang, J., Kong, H., Zeng, Y. and Liu, G., 2018. Functional biomimetic nanoparticles for drug delivery and theranostic applications in cancer treatment. Science and technology of advanced materials, 19(1), pp.771-790. https://doi.org/10.1080/14686996.2018.1528850

[14] Florek, J., Caillard, R. and Kleitz, F., 2017. Evaluation of mesoporous silica nanoparticles for oral drug delivery-current status and perspective of MSNs drug carriers. Nanoscale, 9(40), pp.15252-15277. https://doi.org/10.1039/C7NR05762H

[15] Baeza, A., Ruiz-Molina, D. and Vallet-Regí, M., 2017. Recent advances in porous nanoparticles for drug delivery in antitumoral applications: inorganic nanoparticles and nanoscale metal-organic frameworks. Expert opinion on drug delivery, 14(6), pp.783-796. https://doi.org/10.1080/17425247.2016.1229298

[16] Ibiyeye, K.M., Zuki, A.B.Z., Nordin, N. and Ajat, M., 2019. Ultrastructural Changes and Antitumor Effects of Doxorubicin/Thymoquinone-Loaded $\mathrm{CaCO} 3$ Nanoparticles on Breast Cancer Cell Line. Frontiers in Oncology, 9, p.599. https://doi.org/10.3389/fonc.2019.00599

[17] Sercombe, L., Veerati, T., Moheimani, F., Wu, S.Y., Sood, A.K. and Hua, S., 2015. Advances and challenges of liposome assisted drug delivery. Frontiers in pharmacology, 6, p.286. https://doi.org/10.3389/fphar.2015.00286

[18] Al-Ahmady, Z., Lozano, N., Mei, K.C. and Kostarelos, K., 2016. Engineering thermosensitive liposome-nanoparticle hybrids loaded with doxorubicin for heat-triggered drug release. International Journal of Pharmaceutics, 514(1), pp.133-141 https://doi.org/10.1016/j.ijpharm.2016.09.009

[19] Kanwal, U., Irfan Bukhari, N., Ovais, M., Abass, N., Hussain, K. and Raza, A., 2018. Advances in nano-delivery systems for doxorubicin: an updated insight. Journal of drug targeting, 26(4), pp.296-310. https://doi.org/10.1080/1061186X.2017.1380655

[20] Robson, A.L., Dastoor, P.C., Flynn, J., Palmer, W., Martin, A., Smith, D.W., Woldu, A. and Hua, S., 2018. Advantages and limitations of current imaging techniques for characterizing liposome morphology. Frontiers in pharmacology, 9, p. 80 . https://doi.org/10.3389/fphar.2018.00080

[21] Yingchoncharoen, P., Kalinowski, D.S. and Richardson, D.R., 2016. Lipid-based drug delivery systems in cancer therapy: what is available and what is yet to come. Pharmacological Reviews, 68(3), pp.701-787. https://doi.org/10.1124/pr.115.012070

[22] Kesharwani, S.S., Kaur, S., Tummala, H. and Sangamwar, A.T., 2018. Multifunctional approaches utilizing polymeric micelles to circumvent multidrug resistant tumors. Colloids and Surfaces B: Biointerfaces. https://doi.org/10.1016/j.colsurfb.2018.10.022

[23] Dzmitruk, V., Apartsin, E., Ihnatsyeu-Kachan, A., Abashkin, V., Shcharbin, D. and Bryszewska, M., 2018. Dendrimers Show Promise for siRNA and microRNA Therapeutics. Pharmaceutics, 10(3), p.126. https://doi.org/10.3390/pharmaceutics10030126

[24] Franiak-Pietryga, I., Ziemba, B., Messmer, B. and Skowronska-Krawczyk, D., 2018. Dendrimers as Drug Nanocarriers: The Future of Gene Therapy and Targeted Therapies in Cancer. In Dendrimers-Fundamentals and Applications. IntechOpen. https://doi.org/10.5772/intechopen.75774

[25] Tang, F., Li, L. and Chen, D., 2012. Mesoporous silica nanoparticles: synthesis, biocompatibility and drug delivery. Advanced materials, 24(12), pp.1504-1534. https://doi.org/10.1002/adma.201104763

[26] Paris, J.L., Cabañas, M.V., Manzano, M. and Vallet-Regí, M., 2015. Polymer-grafted mesoporous silica nanoparticles as ultrasound-responsive drug carriers. ACS Nano, 9(11), pp.11023-11033. https://doi.org/10.1021/acsnano.5b04378

[27] Liang, R., Wei, M., Evans, D.G. and Duan, X., 2014. Inorganic nanomaterials for bioimaging, targeted drug delivery and therapeutics. Chemical Communications, 50(91), pp.14071-14081 https://doi.org/10.1039/C4CC03118K

[28] Castillo, R.R., Colilla, M. and Vallet-Regí, M., 2017. Advances in mesoporous silica-based nanocarriers for co-delivery and combination 
therapy against cancer. Expert opinion on drug delivery, 14(2), pp.229-243.

https://doi.org/10.1080/17425247.2016.1211637

[29] Hossen, S., Hossain, M.K., Basher, M.K., Mia, M.N.H., Rahman, M.T. and Uddin, M.J., 2019. Smart nanocarrier-based drug delivery systems for cancer therapy and toxicity studies: A review. Journal of advanced research, 15, pp.1-18. https://doi.org/10.1016/j.jare.2018.06.005

[30] Alexiou, C., Tietze, R., Schreiber, E., Jurgons, R., Richter, H., Trahms, L., Rahn, H., Odenbach, S. and Lyer, S., 2011. Cancer therapy with drug loaded magnetic nanoparticles - magnetic drug targeting. Journal of Magnetism and Magnetic Materials, 323(10), pp.1404-1407. https://doi.org/10.1016/j.jmmm.2010.11.059

[31] Pradhan, P., Jaiswal, M.K., Gogoi, M., Bahadur, D. and Banerjee, R.,2011. Magnetic Nanoparticles and Thermosensitive Carriers for Hyperthermia and Drug Delivery.

[32] Rahban, D., Doostan, M. and Salimi, A., 2020. Cancer therapy; prospects for application of nanoparticles for magnetic-based hyperthermia. Cancer Investigation, (just-accepted), pp.1-28.

[33] Jahanban-Esfahlan, R., Derakhshankhah, H., Haghshenas, B., Massoumi, B., Abbasian, M. and Jaymand, M., 2020. A bio-inspired magnetic natural hydrogel containing gelatin and alginate as a drug delivery system for cancer chemotherapy. International Journal of Biological Macromolecules. https://doi.org/10.1016/j.ijbiomac.2020.04.074

[34] Nguyen, M.P. and Kim, D., 2020. Integration of iron oxide nanoparticles and polyaspartamide biopolymer for MRI image contrast enhancement and an efficient drug-delivery system in cancer therapy. Nanotechnology, 31(33), p.335712. https://doi.org/10.1088/1361-6528/ab8f49

[35] Ayyanaar, S., Balachandran, C., Bhaskar, R.C., Kesavan, M.P., Aoki, S., Raja, R.P., Rajesh, J., Webster, T.J. and Rajagopal, G., 2020. ROS-Responsive Chitosan Coated Magnetic Iron Oxide Nanoparticles as Potential Vehicles for Targeted Drug Delivery in Cancer Therapy. International Journal of Nanomedicine, 15, p.3333. https://doi.org/10.2147/IJN.S249240

[36] Masood, F., 2016. Polymeric nanoparticles for targeted drug delivery system for cancer therapy. Materials Science and Engineering: C, 60, pp.569-578. https://doi.org/10.1016/j.msec.2015.11.067

[37] Frangville, C., Rutkevičius, M., Richter, A.P., Velev, O.D., Stoyanov, S.D. and Paunov, V.N., 2012. Fabrication of environmentally biodegradable lignin nanoparticles. ChemPhysChem, 13(18), p.4235. https://doi.org/10.1002/cphc.201200537

[38] Gao, W. \& Fatehi, P. 2019. Lignin for polymer and nanoparticle production: Current status and challenges. The Canadian Journal of Chemical Engineering, 97, 2827-2842. https://doi.org/10.1002/cjce.23620

[39] Xiong, F., Han, Y., Wang, S., Li, G., Qin, T., Chen, Y. \& Chu, F. 2017. Preparation and Formation Mechanism of Renewable Lignin Hollow Nanospheres with a Single Hole by Self-Assembly. ACS Sustainable Chemistry \& Engineering, 5, 2273-2281. https://doi.org/10.1021/acssuschemeng.6b02585

[40] Tietze, R., Zaloga, J., Unterweger, H., Lyer, S., Friedrich, R.P., Janko, C., Pöttler, M., Dürr, S. and Alexiou, C., 2015. Magnetic nanoparticle-based drug delivery for cancer therapy. Biochemical and biophysical research communications, 468(3), pp.463-470. https://doi.org/10.1016/j.bbrc.2015.08.022

[41] Soetaert, F., Korangath, P., Serantes, D., Fiering, S. and Ivkov, R., 2020. Cancer therapy with iron oxide nanoparticles: Agents of thermal and immune therapies. Advanced Drug Delivery Reviews. https://doi.org/10.1016/j.addr.2020.06.025

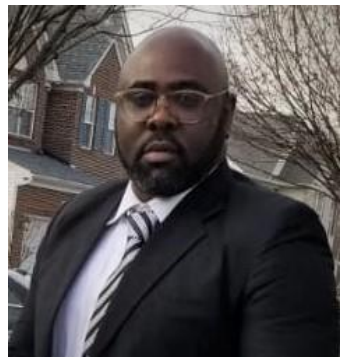

A. John Leo, MSc. obtained a National Diploma and a Bachelor's Degree in Chemistry from The Cape Peninsula University of Technology in 2010 and 2013, respectively. In 2017, he graduated with a Master's degree in Chemistry from The University of Johanessburg. He is currently pursuing a $\mathrm{PhD}$ degree in Chemical Engineering at the North-West University, Potchefstroom, South-Africa in collaboration with Cape Breton University, Nova Scotia, Canada. He has 10 years of working experience in food quality control, mostly working with Freddy Hirsch Group, South Africa as an assistant Laboratory Technician, a Process Controller and a Project Technician. His research interest is in the medical and environmental application of nanotechnology techniques, and currently working on developing of a drug delivery system for cancer therapy. His research work has been useful over the years producing 3 published articles in a peer review journal, including a book chapter. 